\title{
Clonidine or remifentanil for adequate surgical conditions in patients undergoing endoscopic sinus surgery: A randomized study
}

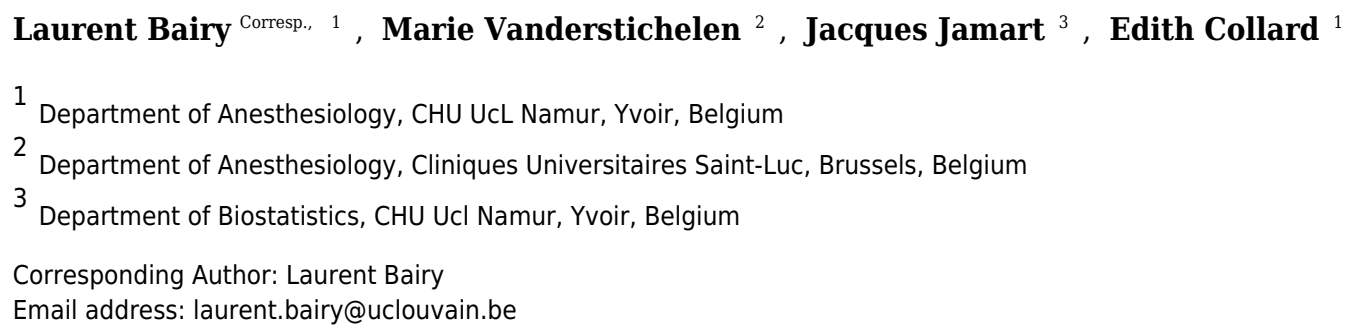

Background. Deliberate hypotension is one way to achieve a bloodless surgical field in endoscopic sinus surgery (ESS). We compared two anaesthesia regimens to induce deliberate hypotension and attempted to determine the most efficient one.

Methods. Fifty-nine patients undergoing ESS were minimized into two groups. In the CLO group, patients received I.V. sufentanil $0.15 \mu \mathrm{g} / \mathrm{kg}$ together with I.V. clonidine $2-3 \mu \mathrm{g} / \mathrm{kg}$. In the REMI group, patients received remifentanil at a rate of up to $1 \mu \mathrm{g} / \mathrm{kg} / \mathrm{min}$. Fromme scores were collected $15 \mathrm{~min}$ after the incision and at the end of the procedure. Mean arterial pressure readings (MAP), heart rate readings, time to eyes opening, time to extubation, pain scores, analgesic requirements, and oxygen needs were collected and compared.

Results. There were no significant differences in Fromme scores between the two groups. The averaged MAP from 15 min to the end of the procedure was significantly lower in the REMI group; these patients also received more ephedrine. Significantly fewer patients in the CLO group needed oxygen therapy to keep their Pulse Oximeter Oxygen Saturation within 3\% of their preoperative values. Patients in this group also needed less piritramide in the recovery room, and their pain scores were lower at discharge from the recovery room.

Discussion. Although both anaesthesia regimens offered a similar quality of surgical field, this study suggests that clonidine had a better average safety profile. Furthermore, patients who received this regimen required fewer painkillers immediately after surgery. 
1 Clonidine or remifentanil for adequate surgical conditions in patients undergoing endoscopic

2 sinus surgery: A randomized study

3 Laurent Bairy ${ }^{1}$, Marie Vanderstichelen ${ }^{2}$, Jacques Jamart ${ }^{3}$, Edith Collard ${ }^{1}$

$4 \quad{ }^{1}$ Department of anesthesiology, CHU UcL Namur, Yvoir, Belgium

$5 \quad{ }^{2}$ Department of anesthesiology, Cliniques Universitaires Saint-Luc, Brussels, Belgium

$6{ }^{3}$ Department of biostatistics, CHU UcL Namur, Yvoir, Belgium

7

8 Corresponding Author:

9 Laurent Bairy ${ }^{1}$

10 
11

12

Abstract

Background. Deliberate hypotension is one way to achieve a bloodless surgical field in endoscopic sinus surgery (ESS). We compared two anaesthesia regimens to induce deliberate hypotension and attempted to determine the most efficient one.

Methods. Fifty-nine patients undergoing ESS were minimized into two groups. In the CLO group, patients received I.V. sufentanil $0.15 \mu \mathrm{g} / \mathrm{kg}$ together with I.V. clonidine $2-3 \mu \mathrm{g} / \mathrm{kg}$. In the REMI group, patients received remifentanil at a rate of up to $1 \mu \mathrm{g} / \mathrm{kg} / \mathrm{min}$. Fromme scores were collected $15 \mathrm{~min}$ after the incision and at the end of the procedure. Mean arterial pressure readings (MAP), heart rate readings, time to eyes opening, time to extubation, pain scores, analgesic requirements, and oxygen needs were collected and compared.

Results. There were no significant differences in Fromme scores between the two groups. The averaged MAP from 15 min to the end of the procedure was significantly lower in the REMI group; these patients also received more ephedrine. Significantly fewer patients in the CLO group needed oxygen therapy to keep their Pulse Oximeter Oxygen Saturation within 3\% of their preoperative values. Patients in this group also needed less piritramide in the recovery room, and their pain scores were lower at discharge from the recovery room.

Discussion. Although both anaesthesia regimens offered a similar quality of surgical field, this study suggests that clonidine had a better average safety profile. Furthermore, patients who received this regimen required fewer painkillers immediately after surgery. 
31 Clonidine or remifentanil for adequate surgical conditions in patients undergoing endoscopic sinus

32 surgery: A randomized study

33

34 Introduction

35 Over the past decades, endoscopic sinus surgery (ESS) has developed into one of the most frequently

36 used surgical treatments for chronic inflammatory or infectious sinus diseases. This procedure requires a

37 bloodless surgical field to help the surgeon visualize anatomical structures to avoid injuries and rare, but

38 potentially catastrophic, complications (Baker \& Baker, 2010).

39 To achieve a bloodless surgical field, deliberate hypotension is often used among other interventions,

40 such as preoperative oral corticosteroid treatment prescribed in case of severe disease, moderate

41 hypocapnia, reverse Trendelenburg position, infiltration with a local anesthetic solution containing a

42 vasopressor and/or topical anesthesia of the surgical site.

43 Deliberate hypotension has been used in numerous procedures to decrease blood loss or increase surgical

44 visibility. There are multiple drugs and techniques that can be used to induce such deliberate hypotension,

45 from anesthesia drugs that have hypotension as side effect (volatile anesthetics, remifentanil) to specific

46 hypotensive agents such as nitroprusside, nicardipine, adenosine, nitroglycerine or esmolol. Some

47 antihypertensive drugs are also used such as ACE inhibitors or clonidine. (Degoutte, 2009)

48 Clonidine is an antihypertensive drug introduced in the 1970s. It is used increasingly in the perioperative

49 setting due to its desirable properties such as morphinic and hypnotic sparing effect, cardioprotection,

50 anxiolysis, reduced shivering, reduced perioperative stress response (Wallace, 2006). Often, it lowers

51 arterial pressure down to values compatible with the definition of controlled hypotension when used in

52 conjunction with volatile anesthetic or propofol. It has already been used in multiple studies related to

53 deliberate hypotension. Remifenanil is an ultrashort acting $\mu$ agonist. It produces consistent hypotension

54 as a side effect. These two drugs are the most used in our institution to induce deliberate hypotension in

55 case of ESS. The aim of this study is to compare two anesthesia regimens that include these drugs and 
56 determine which regimen gives the best operating conditions to the surgeon. Other data collected and

57 compared were emergence duration, pain intensity, hemodynamic and oxygenation $\left(\mathrm{SpO}_{2}\right)$ parameters.

58 In this study, deliberate hypotension is defined by a target mean arterial pressure (MAP) between 55 and

$5965 \mathrm{mmHg}$.

60 Methods

61 Patients

62 All patients with chronic sinus disease, older than 18 years, categorized ASA physical status I to III,

63 primary or secondary surgery were assessed for eligibility. Exclusion criteria included cardiac disorders

64 other than supraventricular tachycardia, cerebrovascular disorders, renal or hepatic disorders, non-treated

65 arterial hypertension, beta-blocking agent therapy, platelet-inhibiting agent or anticoagulant therapy,

66 coagulopathy, and pregnancy. Fifty-nine patients were enrolled in the study between November 2014 and

67 March 2015 at the CHU UcL Namur, a tertiary care center in Belgium. Patients were prospectively

68 randomized in the CLO group or the REMI group using minimization (Saghaei M \& Saghaei S, 2011) the

69 day before or the day of the surgery by the main investigator (figure 1). The factors used for minimization

70 included age, sex, Mackay-Lund score that assesses the extent of the sinus disease on a sinus CT scan

71 (Fokkens, Lund \& Mullol 2007), preoperative oral corticoid treatment, ASA score and one of the two

72 possible surgeons.

73 Treatment

74 In the CLO group, anesthesia was induced with clonidine $2 \mu \mathrm{g} / \mathrm{kg}$ slow I.V. bolus over $10 \mathrm{~min}$, sufentanil

$750.15 \mu \mathrm{g} / \mathrm{kg}$, propofol titrated to loss of eyelid reflex and rocuronium $0.3 \mathrm{mg} / \mathrm{kg}$. In the REMI group,

76 anesthesia was induced with a remifentanil I.V. infusion of $0.25 \mu \mathrm{g} / \mathrm{kg} / \mathrm{min}$, propofol titrated to the loss of

77 eyelid reflex, and rocuronium $0.3 \mathrm{mg} / \mathrm{kg}$. The remifentanil infusion rate was halved after tracheal

78 intubation. In both groups, the trachea was intubated and the anesthesia was maintained using sevoflurane

$791 \mathrm{MAC}$ (age corrected) in a $50 \% \mathrm{FiO}_{2}$ oxygen/air mixture. The gas mixture was delivered to the patient

80 by a Dräger Zeus ${ }^{\mathrm{TM}}$ (Dräger Medical, Lübeck, Germany) ventilator in a circle circuit using the "auto-

81 controlled mode". In both groups, the following settings were used for mechanical ventilation: $3 \mathrm{~cm} \mathrm{H}_{2} \mathrm{O}$ 
82 PEEP , 6-8 $\mathrm{ml} / \mathrm{kg}$ tidal volume and respiratory rate of $12 / \mathrm{min}$. These parameters were subsequently

83 adjusted to maintain $\mathrm{EtCO}_{2}$ between 30 and $35 \mathrm{mmHg}$. The patients were positioned $15^{\circ}$ reverse

84 Trendelenburg. Non-invasive blood pressure, heart rate (HR), transcutaneous oxygen saturation $\left(\mathrm{SpO}_{2}\right)$

85 and train of four (TOF) were measured using a Philips MX800 monitor (Phillips Medical, Nijmegen, the

86 Netherlands). These parameters, along with respiratory parameters from the ventilator, were recorded by

87 the Exacto ${ }^{\mathrm{TM}}$ computerized anesthesia record system (Mexys, Mons, Belgium). Local infiltration with

88 lidocaine $1 \%$ and epinephrine 1/200000 was performed and followed by a topical application of lidocaine

89 and naphazoline for mucosal anesthesia and vasoconstriction. If the surgeon complained about the

90 bleeding or if the Fromme score (Fromme et al., 1986) (Table 1) was 3, 4 or 5 and the MAP was higher

91 than $65 \mathrm{mmHg}$, the patients in the CLO group received an additional $1 \mu \mathrm{g} / \mathrm{kg}$ slow I.V. clonidine bolus

92 (up to a maximum total dose of $3 \mu \mathrm{g} / \mathrm{kg}$ ) or a sufentanil $5 \mu \mathrm{g} \mathrm{I} . \mathrm{V}$. bolus if the previous one was

93 administered more than 45 min before. In the REMI group, the remifentanil infusion rate was doubled up

94 to a maximum rate of $1 \mu \mathrm{g} / \mathrm{kg} / \mathrm{min}$. Despite these measures, if the surgeon still noted considerable

95 bleeding, the patient was excluded from the study.

96 If the MAP was lower than $55 \mathrm{mmHg}$, ephedrine was titrated in 3-mg increments to achieve a MAP

97 higher than $55 \mathrm{mmHg}$. Additionally, in the REMI group, the remifentanil infusion rate was halved. In

98 case of failure to maintain a MAP higher to $55 \mathrm{mmHg}$ using ephedrine, the patient was excluded from the 99 study.

100 Sevoflurane and remifentanil, if applicable, were stopped when the surgeon began packing the nose and

101 the patient's TOF ratio attained 0.9. Patients who had still a TOF ratio lower than 0.9 were given

102 glycopyrrolate $0.5 \mathrm{mg}$ and neostigmine $2.5 \mathrm{mg}$. Analgesia was provided by giving the patient paracetamol

$1031 \mathrm{~g} \mathrm{I.V.,} \mathrm{tramadol} 100 \mathrm{mg}$ I.V. and ketorolac $30 \mathrm{mg} \mathrm{I.V.,} \mathrm{respecting} \mathrm{contraindications} \mathrm{and} \mathrm{dosage}$

104 limitations for each patient, if appropriate. All patients received $50 \mathrm{mg}$ alizapride I.V. to prevent nausea

105 and vomiting. In the recovery room, oxygen was delivered through a facemask to keep the patient's $\mathrm{SpO}_{2}$

106 within $3 \%$ of the preoperative value. 
107 The patient was discharged from the recovery room when his/her modified Aldrete score was $\geq 9$ and

108 when the patient was comfortable.

109

110 Assessment

111 We decided not to use blood loss as an indicator, as it is very difficult to measure accurately due to the

112 large amount of irrigation fluid used in this type of surgery and, more often than not, it is minimal. Blood

113 loss was grossly estimated to ensure that the patient did not reach the transfusion threshold.

114 The quality of the surgical field was assessed by the surgeon (blinded), using the Fromme score 15

115 minutes after the start of the surgery (From15) to allow the MAP and HR reach an equilibrium after the

116 local anesthetic infiltration and the first surgical stimulus. The Fromme score at the end of the surgery

117 (FromE) gave an overall rating of the surgical field quality. The quality of the surgical field was

118 characterized as low when the Fromme score was higher than 2 or high if the score was lower or equal to

1192.

120 In the recovery room, pain was evaluated using the 11-point numerical rating scale (NRS-11), which is a

121 patient self-reported pain score that ranges from 0 implying no pain to 10 implying the worst pain

122 imaginable. If the patient reported a NRS-11 higher than 3, piritramide was administered in $2 \mathrm{mg}$

123 increments.

124 The following parameters were also recorded and compared: continuous $\mathrm{SpO}_{2}$ and $\mathrm{HR}$, MAP every 3 min

125 during the surgery and every $10 \mathrm{~min}$ in the recovery room, time to eyes opening (time patient opened

$126 \mathrm{his} /$ her eyes- time when sevoflurane $+/$ - remifentanil was/were stopped), time to extubation (time patient

127 was extubated - time sevoflurane +/- remifentanil was/were stopped), time spent in the recovery room,

128 pain scores at the arrival and departure from the recovery room, piritramide consumption in the recovery

129 room and need for oxygen when patient returned to the ward.

130 Averaged MAP readings (MAPA) and averaged HR (HRA) values from 15 min after the onset of the

131 procedure to the end of the surgery were computed using the computerized anesthesia record system

132 database. 
133 Statistics

134 The sample size was computed to allow detection of a difference between Fromme scores of 1 point, with

135 a standard deviation of 1.3 estimated from a preliminary assessment of the Fromme score on 40 patients,

136 with an $\alpha$ level of 0.05 and a power $(1-\beta)$ of 0.80 . The computation used $G^{*}$ Power 3 software (Heinrich

137 Heine Universität, Dusseldorf, Germany) (Faul et al., 2007) and led to the total sample size of 58 patients

138 All the analyses were performed with the intention to treat.

139 Statistical analyses were carried out using SPSS ${ }^{\text {TM }}$ software (v18.0, SPSS Inc., Chicago, IL, USA).

140 As we were not able to claim the normality of all numerical variables, their comparisons were performed

141 using the Wilcoxon-Mann-Whitney test, and categorical variables were compared using Fisher's exact

142 test. A $p$-value of 0.05 was considered significant and was not corrected for the number of tests

143 performed.

144

145 Results

146 No patient was excluded from the study.

147 There were no significant differences between both groups in regard to body mass index (BMI),

148 preoperative MAP, $\mathrm{HR}, \mathrm{SpO}_{2}$ values, and duration of surgery. In the CLO group, 36.7\% were smokers

149 compared to $6.9 \%$ in the REMI group $(p=0.01)$ (Table 2$)$.

150 In the CLO group, 20 patients received clonidine $2 \mu \mathrm{g} / \mathrm{kg} \mathrm{I.V}$. and 9 patients received $3 \mu \mathrm{g} / \mathrm{kg} \mathrm{I.V}$. The

151 mean \pm SD sufentanil dose was $0.16 \mu \mathrm{g} / \mathrm{kg} \pm 0.03 \mu \mathrm{g} / \mathrm{kg}$. In the REMI group, patients received 0.34

$152 \mu \mathrm{g} / \mathrm{kg} / \mathrm{min} \pm 0.17 \mu \mathrm{g} / \mathrm{kg} / \mathrm{min}($ mean $\pm \mathrm{SD})$ of remifentanil.

153 There were no significant differences in the From 15 or FromE scores between the two groups (Table 3).

154 The quality of the surgical field was deemed low in 9 patients in the CLO group compared to 11 patients

155 in the REMI group at $15 \mathrm{~min}(\mathrm{p}=0.59)$ and 16 patients in the CLO group compared to 10 patients in the

156 REMI group at the end of the surgery $(\mathrm{p}=0.19)$.

157 The MAP and HR at 15 min after incision were similar in both groups. HRA did not differ between the

158 two groups. The MAPA was significantly lower in the REMI group. The total amount of ephedrine 
159 administered to the patients was significantly higher in the REMI group, as $79.3 \%$ of these patients

160 needed ephedrine (on average $11 \mathrm{mg}$ to maintain their MAP in the admissible range). In the CLO group,

$16146.6 \%$ of the patients needed ephedrine (on average $5.9 \mathrm{mg}$ ) (Table 3).

162 Time to eye opening, time to extubation, NRS-11 at arrival in the recovery room and time spent in the

163 recovery room did not significantly differ between the two groups. The pain score at discharge from the

164 recovery room was significantly lower in the CLO group, as was the piritramide consumption. The

165 patients from the REMI group had a significantly higher probability to be discharged from the recovery

166 room with supplemental oxygen to maintain their $\mathrm{SpO}_{2}$ within 3\% from their preoperative values (Tables

1673 and 4).

168 There was no difference in supplemental oxygen at departure from the recovery room between smokers

169 and non-smokers $(p=0.67)$ or patients having a BMI $<=25$ and $>25(p=0.12)$.

170 Upon arrival in the recovery room, one patient in the CLO group developed a hypotension (MAP of 60

$171 \mathrm{mmHg}$ ) that was successfully treated with a fluid bolus, and one patient in the REMI group developed a

172 sinus tachycardia (HR 120-140/min) that spontaneously ceased after 1 hour. No patient suffered from

173 nausea or vomiting, bradycardia, hypertension, and other unwanted side effects. 
174 Discussion

175 Many factors are involved in the quality of the surgical field in ESS (e.g., anatomical conditions and

176 severity and inflammatory status of the disease). In this study, these factors have been considered by

177 including the CT-based Mackay-Lund score, a known predictor of bleeding in ESS (Mortuaire et al.,

178 2008), and preoperative oral corticoid treatment in the factors used for the minimization. The anesthetic

179 technique also has an impact on the surgeon's comfort. Patient positioning, local anesthetic infiltration,

180 respiratory parameters, and even the way the ETT is secured have been shown to affect blood loss (Baker

$181 \&$ Baker, 2010).

182 Both clonidine and remifentanil have been used and studied in the setting of controlled hypotension

183 (Wawrzyniak, Kusza \& Cywinski, 2014; Miłoński et al., 2013; Mohseni \& Ebneshahidi, 2011; Marchal et

184 al., 2001; Lee et al., 1999; Hackmann et al., 2003; Yun, Kim \& Kim, 2015; Cardesin et al., 2015). These

185 drugs decrease blood loss by decreasing the blood pressure and, more specifically, the cardiac output.

186 Kazmeier et al. found that remifentanil lowers stroke volume and HR in similar proportions, decreasing

187 cardiac output and MAP (Kazmeier et al., 2000). Clonidine also decreases blood pressure by lowering

188 cardiac output. Its action is mainly due to the decrease in HR, leaving the stroke volume and the systemic

189 vascular resistance untouched (Onesti et al., 1971). I.V. clonidine can produce a transient hypertensive

190 effect secondary to systemic vasoconstriction, which was not encountered in our study due to the slow

191 speed of the I.V. injection.

192 In the present study, we showed that both drugs provided the surgeon similar operating conditions with a

193 slightly lower MAP in the REMI group. Ephedrine requirements were significantly higher and more

194 frequent in this group than in the CLO group. One could argue that the remifentanil initial infusion rate

195 was too high. We do not agree with this argument, as the rate was adjusted by a factor of 2 according to

196 the MAP.

197 The longer half-life of clonidine and sufentanil in comparison to remifentanil as well as the sedative

198 properties of the alpha-2 agonist did not affect the recovery times. Time to eyes opening, time to

199 extubation, and length of stay in the recovery room were not significantly different between both groups. 
200 Time to eyes opening and extubation times might seem long, but all anesthetic drug administration was

201 stopped when the surgeon began packing the nose at the very end of the procedure. The study protocol

202 was strict regarding when and how drug administration could be changed. There were no fixed rules for

203 the extubation criteria, which left a certain degree of freedom to the anesthetist in charge.

204 Patients who received clonidine were less prone to require supplemental oxygen to maintain their $\mathrm{SpO}_{2}$

205 within $3 \%$ of their preoperative values. Clonidine is a drug that has shown a positive effect on the overall

206 saturation on patients suffering from obstructive sleep apnea syndrome (OSAS). This effect was first

207 thought to be the result of the drug's ability to decrease or suppress time spent in REM sleep (Issa, 1992).

208 Clonidine has since been studied as a premedication in patients with OSAS and has shown a higher

209 minimal SpO2 on the day of surgery in patients who received clonidine versus placebo (Pawlik et al.,

210 2005). The authors explain this difference by the lower amount of propofol and piritramide received by

211 patients with clonidine premedication. In our study, no such difference was observed. The piritramide

212 consumption is statistically different between the two groups but clinically not relevant, and remifentanil

213 did not affect ventilatory mechanics after more than an hour spent in the recovery room. Clonidine is

214 known to decrease the ventilatory response to $\mathrm{CO}_{2}$, which is seen as a central depressant effect by Ooi et

215 al. in 1991, but is considered a protective mechanism against central hypocapnic apnea during non-REM

216 sleep by Sankr-Tarbichi, Grullon \& Badr (2013). It remains unclear how clonidine could have a positive

217 effect on awake patients' $\mathrm{SpO}_{2}$ following ESS. We recommend further studies to address this issue. In

218 this study, we did not find a relationship between supplemental oxygen and smoking status or the

219 patient's BMI.

220 NRS-11 were similar in both groups upon arrival in the recovery room, but were slightly higher in the

221 REMI group upon discharge. Although this difference is statistically significant, it is probably not

222 clinically relevant. Nevertheless, it should be noted that more patients in the REMI group received

223 piritramide at a higher dose than in the CLO group. These results concur with previous results of studies

224 showing that clonidine potentiates opioid drugs or has intrinsic analgesic properties (Blaudszun et al., 
225 2012; Wallace, 2006). These results could also be explained by the longer acting sufentanil used in the 226 CLO group.

227 In contrast to Cardesin et al. (2015) we did not find an advantage of clonidine over remifentanil for ESS

228 in regard to the surgical field quality. Their anesthesia protocol was slightly different, with lower doses of

229 hypotensive agent (clonidine $2-3 \mu \mathrm{g} / \mathrm{kg}$ vs. $1-1.5 \mu \mathrm{g} / \mathrm{kg}$ and remifentanil $0.34 \mu \mathrm{g} / \mathrm{kg} / \mathrm{min}$ vs. 0.1-0.2

$230 \mu \mathrm{g} / \mathrm{kg} / \mathrm{min}$ ). Cardesin et al. used fentanyl in both group, but we used only sufentanil in the CLO group,

231 which is more rational from a pharmacological point of view and closer to our daily practice. The scores

232 used were different, although loosely comparable as they are both 6-point scales. They were also assessed

233 at different instants. The Boezaart scale is probably less subjective than the Fromme score we used as it is

234 mainly based on the suction usage. Patients and surgical treatments were also different as shown by the

235 Mackay-Lund scores and the mean duration of the surgery. All these elements make it difficult to

236 compare both studies.

237 Conclusions

238 Although both anesthesia regimens provided similar operating conditions, patients who received clonidine 239 and sufentanil had significantly higher MAP, required less vasopressor, received less piritramide, and had

240 lower NRS-11 scores. Fewer patients in this group needed oxygen to maintain their $\mathrm{SpO}_{2}$ to the

241 preoperative level. The clonidine-sufentanil association has slight advantages, including ease of use, over

242 remifentanil to achieve satisfying operating conditions for patients undergoing ESS for chronic

243 rhinosinusitis.

Ethical Statement

246 This study was approved by the local ethics committee (id 101/2003. Belgian unique id:

247 B039201318956). It was prospectively registered in the Australian and New Zealand clinical trial register 248 under the id ACTRN12614000935639. A written informed consent was obtained from all the patients. 
250

251

252

253

254

255

256

257

258

259

260

261

262

263

264

265

266

267

268

269

270

271

272

273

\section{References}

- Baker AR, Baker AB. 2010. Anaesthesia for endoscopic sinus surgery. Acta Anaesthesiologica Scandinavica. 54:795-803. DOI: 10.1111/j.1399-6576.2010.02259.x

- Blaudszun G, Lysakowski C, Elia N, Tramèr MR. 2012. Effect of perioperative systemic $\alpha 2$ agonists on postoperative morphine consumption and pain intensity: systematic review and metaanalysis of randomized controlled trials. Anesthesiology. 116(6):1312-22. DOI:

\subsection{7/ALN.0b013e31825681cb}

- Cardesin A, Pontes C, Rosell R, Escamilla Y, Marco J, Escobar MJ, Bernal-Sprekelsen M. 2015. A randomised double blind clinical trial to compare surgical field bleeding during endoscopic sinus surgery with clonidine-based or remifentanil-based hypotensive anaesthesia. Rhinology. 53(2):107-15. DOI: 10.4193/Rhin14.185

- Faul F, Erdfelder E, Lang A-G, Buchner A. 2007. G*Power 3 A flexible statistical power analysis program for the social, behavioral, and biomedical sciences. Behavior Research Methods. 39:175191. DOI: $10.3758 / \mathrm{BF} 03193146$

- Fokkens W, Lund V, Mullol J. 2007. European Position Paper on Rhinosinusitis and Nasal Polyps group. Rhinology. 20:1-136.

- Fromme GA, MacKenzie RA, Gould AB Jr, Lund BA, Offord KP. 1986. Controlled hypotension for orthognathic surgery. Anesthesia \& Analgesia. 65:683-6.

- Hackmann T, Friesen M, Allen S, Precious DS. 2003. Clonidine facilitates controlled hypotension in adolescent children. Anesthesia \& Analgesia. 96(4):976-81. DOI: 10.1213/01.ANE.0000052713.72875.13

- Issa, F.G., 1992. Effect of Clonidine in Obstructive Sleep Apnea1 • 2. Am Rev Respir Dis, 145, pp.435-439. DOI: $10.1164 /$ ajrccm/145.2_Pt_1.435 
- Kazmaier S, Hanekop GG, Buhre W, Weyland A, Busch T, Radke OC, Zoelffel R, Sonntag H. 2000. Myocardial consequences of remifentanil in patients with coronary artery disease. British Journal of Anaesthesia. 84(5):578-83. DOI: 10.1093/bja/84.5.578

- Lee J, Lovell AT, Parry MG, Glaisyer HR, Bromley LM. 1999. I.v. clonidine: does it work as a hypotensive agent with inhalation anaesthesia?. British Journal of Anaesthesia. 82(4):639-40. DOI: $10.1093 / \mathrm{bja} / 82.4 .639$

- Marchal JM, Gómez-Luque A, Martos-Crespo F, Sánchez De La Cuesta F, Martínez-López MC, Delgado-Martinez AD. 2001. Clonidine decreases intraoperative bleeding in middle ear microsurgery. Acta Anaesthesiologica Scandinavica. 45(5):627-33. DOI: 10.1034/j.13996576.2001.045005627.x

- Miłoński J, Zielińska-Bliźniewska H, Golusiński W, Urbaniak J, Sobański R, Olszewski J. 2013.

- Mohseni M, Ebneshahidi A. 2011. The effect of oral clonidine premedication on blood loss and the quality of the surgical field during endoscopic sinus surgery: a placebo-controlled clinical trial. Journal of Anesthesia. 25(4):614-7. DOI: 10.1007/s00540-011-1157-9

- Mortuaire G, Bahij J, Maetz B, Chevalier D. 2008. Lund-Mackay score is predictive of bleeding in ethmoidectomy for nasal polyposis. Rhinology. 46(4):285-8.

- Onesti G, Schwartz AB, Kim KE, Paz-Martinez V, Swartz C. 1971. Antihypertensive effect of clonidine. Circulation Research. 28(5):53-69.

- Ooi, R., Pattison, J. and Feldman, S.A., 1991. The effects of intravenous clonidine on ventilation. Anaesthesia, 46(8), pp.632-633. DOI: 10.1111/j.1365-2044.1991.tb09709.x

- Pawlik, M.T., Hansen, E., Waldhauser, D., Selig, C. and Kuehnel, T.S., 2005. Clonidine premedication in patients with sleep apnea syndrome: a randomized, double-blind, placebo- 
controlled study. Anesthesia \& Analgesia, 101(5), pp.1374-1380.DOI:

300

301

302

303

304

305

306

307

308

309
10.1213/01.ANE.0000180194.30741.40

- Saghaei M, Saghaei S. 2011. Implementation of an open-source customizable minimization program for allocation of patients to parallel groups in clinical trials. Journal of Biomedical Science and Engineering.4, 734-739. DOI: 10.4236/jbise.2011.411090

- Sankri-Tarbichi, A.G., Grullon, K. and Badr, M.S., 2013. Effects of clonidine on breathing during sleep and susceptibility to central apnoea. Respiratory physiology \& neurobiology, 185(2), pp.356-361.DOI:10.1016/j.resp.2012.09.005

- Wallace AW.. Clonidine and modification of perioperative outcome. 2006. Current Opinion in Anaesthesiology. 19(4):411-7. DOI: 10.1097/01.aco.0000236141.15852.0b

- Wawrzyniak K, Kusza K, Cywinski JB. 2014. Comparison of clonidine and midazolam premedication before endoscopic sinus surgery: results of clinical trial. Clinical and Experimental Otorhinolaryngology. 7(4):307-11. DOI: 10.3342/ceo.2014.7.4.307

- Yun SH, Kim JH, Kim HJ. 2015. Comparison of the hemodynamic effects of nitroprusside and remifentanil for controlled hypotension during endoscopic sinus surgery. Journal of Anesthesia. 29(1):35-9. DOI: 10.1007/s00540-014-1856-0 
317 Figure 1. CONSORT flow diagram

318

319

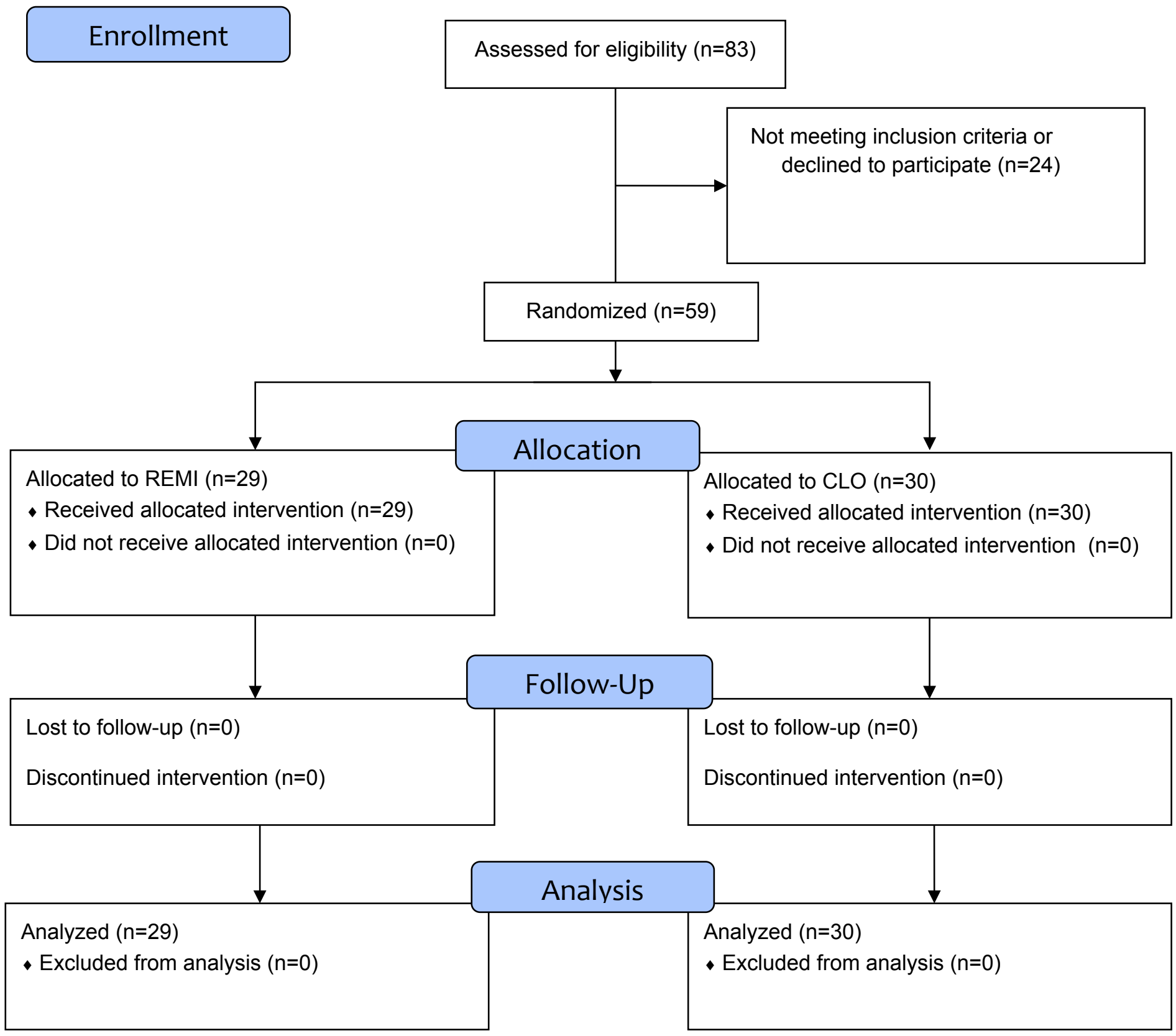




\section{Table 1. Fromme score}

Fromme score

5 Massive uncontrollable bleeding.

4 Bleeding, heavy but controllable, that significantly interfered with dissection.

3 Moderate bleeding that moderately compromised surgical dissection.

2 Moderate bleeding, a nuisance but without interference with accurate dissection.

1 Bleeding, so mild it was not even a surgical nuisance.

0 No bleeding, virtually bloodless field.

321 
323 Table 2. Patients' demographic data

\begin{tabular}{lccc} 
& CLO group $(\mathrm{n}=30)$ & REMI group $(\mathrm{n}=29)$ & $p$ \\
\hline Age (mean $\pm \mathrm{SD})$ & $46 \pm 15$ & $46 \pm 15$ & \\
Sex M/F & $13 / 17$ & $13 / 16$ & \\
Surgeon A/B & $10 / 20$ & $11 / 18$ & \\
ASA I/II/III & $6 / 24 / 0$ & $3 / 25 / 1$ & \\
Preop. oral corticoids & $6.7 \%$ & $13.8 \%$ & 0.39 \\
Mackay-Lund score (median $\pm \mathrm{IQR})$ & $7.4 \pm 5.1$ & $7.2 \pm 5.9$ & $0.01^{*}$ \\
BMI (mean \pm SD) & $25.9 \pm 4.7$ & $27.1 \pm 5.4$ & $6.9 \%$
\end{tabular}

$324 *$ Statistically significant 
325 Table 3. Outcomes expressed as median and interquartile range (IQR) (Wilcoxon-Mann-Whitney test).

\begin{tabular}{|c|c|c|c|c|c|}
\hline & \multicolumn{2}{|c|}{ CLO group } & \multicolumn{2}{|c|}{ REMI group } & \multirow[t]{2}{*}{$p$} \\
\hline & Median & IQR & Median & IQR & \\
\hline From 15 score & 2 & 1.75 & 2 & 1 & 0.58 \\
\hline FromE score & 3 & 1 & 2 & 1 & 0.24 \\
\hline Pain NRS at recovery room admission & 0 & 0 & 0 & 0 & 0.21 \\
\hline Pain NRS at recovery room discharge & 0 & 1 & 1 & 2 & $0.04 *$ \\
\hline Preoperative $\mathrm{SpO}_{2}(\%)$ & 100 & 1 & 100 & 3 & 0.64 \\
\hline Preoperative MAP (mmHg) & 87.5 & 17.5 & 90 & 19 & 0.50 \\
\hline Preoperative HR (bpm) & 67.5 & 13 & 73 & 16 & 0.19 \\
\hline MAP at $15 \mathrm{~min}(\mathrm{mmHg})$ & 61 & 14.75 & 56 & 10 & 0.10 \\
\hline HR at $15 \min (b p m)$ & 68 & 21.75 & 62 & 18 & 0.12 \\
\hline \multicolumn{6}{|l|}{ Mean MAP from 15 min after starting to } \\
\hline end of surgery $(\mathrm{mmHg})$ & 61.5 & 8.5 & 57 & 8 & $0.04 *$ \\
\hline \multicolumn{6}{|l|}{ Mean HR from 15 min after starting to end } \\
\hline of surgery $(\mathrm{mmHg})$ & 64.5 & 18.25 & 64 & 12 & 0.35 \\
\hline Ephedrine total dose (mg) & 0 & 11.25 & 9 & 15 & $0.01 *$ \\
\hline Length of surgery (min) & 53 & 39 & 57 & 41 & 0.63 \\
\hline Time to eyes opening (min) & 16 & 8 & 14 & 8 & 0.43 \\
\hline Time to extubation (min) & 10 & 7 & 13 & 6 & 0.64 \\
\hline Recovery room stay length (min) & 74 & 24 & 76 & 33 & 0.66 \\
\hline Piritramide dose in the recovery room $(\mathrm{mg})$ & 0 & 0 & 0 & 4 & $0.02 *$ \\
\hline Mean $\mathrm{SpO}_{2}$ in the recovery room (\%) & 98 & 2 & 98 & 2 & 0.21 \\
\hline
\end{tabular}

$326 *$ Statistically significant. 
328 Table 4. Percentage of patients who received oxygen and piritramide (Fisher's exact test).

\begin{tabular}{lccc} 
& CLO group & REMI group & $P$ \\
\hline Number of patients discharged from recovery with oxygen & $3.3 \%$ & $24.1 \%$ & $0.03 *$ \\
Number of patients who received piritramide & $10.0 \%$ & $34.5 \%$ & $0.03 *$
\end{tabular}

$329 *$ Statistically significant.

330 